\title{
Comparing Color Doppler Ultrasonography and Angiography to Assess Traumatic Arterial Injuries of the Extremities
}

\author{
Masoud Pezeshki Rad ${ }^{1}$; Mahyar Mohammadifard ${ }^{2, *}$; Hassan Ravari ${ }^{1}$; Donya Farrokh ${ }^{1}$; Emad \\ Ansaripour ${ }^{1}$; Elena Saremi ${ }^{1}$ \\ ${ }^{1}$ School of Medicine, Vascular and Endovascular Research Center, Imam Reza Hospital, Mashhad University of Medical Sciences, Mashhad, Iran \\ ${ }^{2}$ School of Medicine, Vascular and Endovascular Research Center, Imam Reza Hospital, Birjand University of Medical Sciences, Birjand, Iran \\ ${ }^{*}$ Corresponding author: Mahyar Mohammadifard, School of Medicine, Vascular and Endovascular Research Center, Imam Reza Hospital, Birjand University of Medical Sciences, \\ Birjand, Iran. Tel: +98-5118414499, Fax: +98-5612226898, E-mail: mahyar.mohammadifard@yahoo.com \\ Received: August 16, 2013; Revised: April 20, 2014; Accepted: June 1, 2014
}

\begin{abstract}
Background:Traumatic events are one of the major causes of arterial injuries. Physical examination is not a good predictor of the extent of injuries and arteriography is considered as the gold standard for this purpose. In the recent years, noninvasive modalities are increasingly replacing diagnostic arteriography. Color Doppler ultrasonography (USG) is an excellent method to investigate arterial diseases.

Objectives: The present study aimed to evaluate the diagnostic value of color Doppler USG compared to conventional angiography in traumatic arterial injuries of extremities.

Patients and Methods: Seventy-five patients with extremity trauma suspicious for arterial injury were examined by color Doppler USG just before angiography. Doppler pattern and flow states were assessed, then angiography was performed. The results of duplex USG were compared with angiography.

Results: Color Doppler USG had a sensitivity of $95 \%$ and specificity of $98 \%$ in diagnosis of arterial injury. Positive and negative predictive values of Doppler USG were 92.5\% and 94.2\%, respectively.

Conclusion: Color Doppler USG can be used as a reliable modality with acceptable sensitivity and specificity values to screen hemodynamically stable patients with limb trauma suspicious for arterial injury.
\end{abstract}

Keywords: Color Doppler Ultrasonography; Trauma; Angiography; Blood Vessels

\section{Background}

Injuries and accidents are the main causes of morbidity, limb amputation and mortality worldwide. Many of these complications are due to vascular traumas. These injuries need prompt intervention and management due to limited time of tissue viability (1). If hard signs of vascular injuries (i.e. pulsatile hemorrhage, expansile hematoma, palpable thrill and pulselessness) exist, an urgent surgical intervention would be needed (2).

There are some cases in which surgeon cannot precisely judge the condition due to coexistence of other injuries and lack of classic clinical signs (3). In such instances, which are not rare, angiography has been recommended as the standard diagnostic modality. Although its sensitivity and specificity values for detecting vascular injuries have been reported as $95 \%$ to $100 \%$ and $90 \%$ to $98 \%$, respectively, it is an aggressive and expensive modality, which needs special conditions $(4,5)$. Nonetheless, among these suspicious cases, the results of angiographies have been normal or near normal and even a small defect might be noted, which would not change the management course of such cases with trauma (5). Hence, many have tried to substitute angiography with noninvasive modalities such as serial physical examination alone or in combination with other methods including ankle brachial index (ABI), arterial pressure index (API) or Doppler ultrasonography (USG) (6). Color Doppler USG is a noninvasive method widely used for the diagnosis of vascular diseases because of its acceptable accuracy in detection of vascular lesions (7).

\section{Objectives}

In this study, we compared the accuracy of color Doppler USG in the diagnosis of traumatic vascular injuries with that of angiography.

\section{Patients and Methods}

This study was performed in one year and included 75 patients with probable arterial injury in extremities referred to the radiology department of Imam Reza Hospital to undergo angiography. All patients underwent a color Doppler USG examination just before angiography. Examination was performed while the patient was prepared for angiography to prevent wasting time in trauma management. 
We used a Siemens G40 color Doppler USG (Germany) device with a 6- to $10-\mathrm{MHz}$ linear vascular probe. Color Doppler USG was performed by an expert radiologist in vascular USG mode. Angiography reports were written by another radiologist unaware of Doppler examination findings.

depending on the site of injury, blood flow condition inside the artery and Doppler waveform patterns were recorded on three distinct points as follows: on ulnar and radial arteries in wrist, brachial artery in antecubital fossa, and axillary artery in axillary fossa for upper limb; and on anterior and posterior tibial arteries in ankle, popliteal artery in popliteal fossa and common and superficial femoral arteries in inguinal region of the lower limb. In the presence of any change in normal Doppler pattern, all the artery length was evaluated carefully as possible.
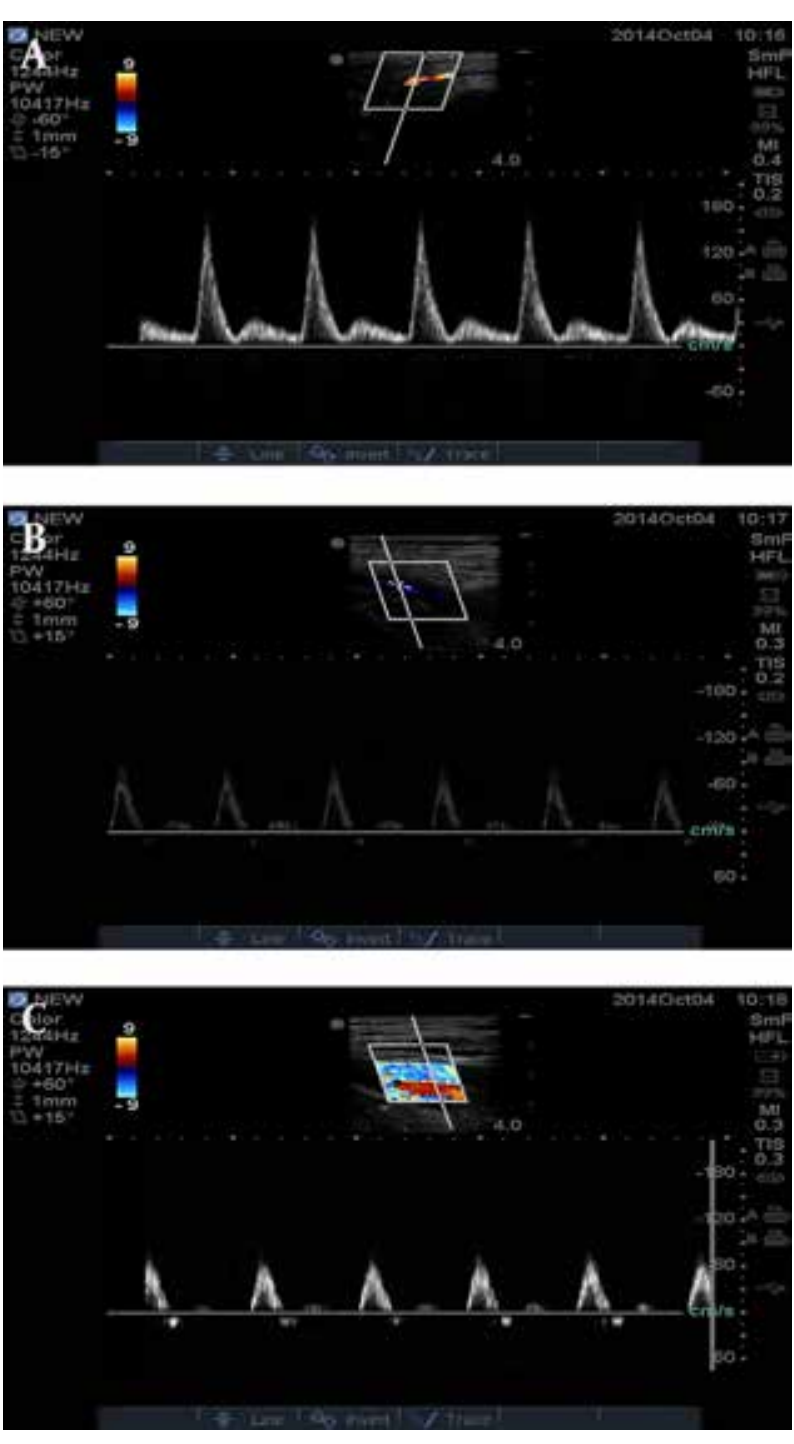

Figure 1. Normal triphasic doppler waves. A, Common femoral artery. B, Popliteal artery. C, Anterior tibial artery.
Normal Doppler study wave was defined as a triphasic pattern in all examination points (Figure 1). This triphasic pattern has three components: rapid antegrade flow with a peak in systole, early diastolic reversal of flow and slow antegrade flow at late diastole. Any other patterns such as damping were considered as abnormal.

After performing angiography, normal finding on angiography was defined as a full column linear and regular intraluminal contrast pattern. Abnormal findings were defined as any interruption of the normal continuity or irregularity of the intraluminal borders (e.g. arterial emboli). Data was analyzed using SPSS ver. 11.5 (SPSS Inc. Chicago, IL, USA). P value below 0.05 was considered statistically significant.

\section{Results}

Eleven of 75 cases were female (14.7\%) and 64 (85.3\%) were male. Fifty-eight patients (77.3\%) had lower limb trauma, while upper limb trauma was seen in 17 patients (22.7\%). The most common cause of trauma was motor vehicle accidents (77.3\%) (Table 1). Thirty-six patients (48\%) had normal angiography findings, while evidences of arterial injury were noticed in 39 patients (52\%).

Doppler ultrasound assessments showed a normal pattern in 35 cases (46.7\%) and abnormal findings in 40 ones (53.3\%) (Table 2 ).

Considering angiography as the gold standard diagnostic tool for arterial injuries, our findings showed a sensitivity of $94.9 \%$ and a specificity of $91.7 \%$ for Doppler USG. Positive and negative predictive values of Doppler USG were $92.5 \%$ and $94.3 \%$, respectively. Patients with abnormal findings on Doppler USG were carefully evaluated for

\begin{tabular}{lccc}
\hline \multicolumn{4}{l}{ Table 1. Mechanism of Trauma in Patients } \\
\hline Mechanism & Male & Female & Total $^{\text {a }}$ \\
\hline Stabbings & 7 & 1 & $8(10.7)$ \\
Gun Shots & 4 & 1 & $5(6.7)$ \\
$\begin{array}{l}\text { Motor Vehicle } \\
\text { Accidents }\end{array}$ & 49 & 9 & $58(77.3)$ \\
Work Accidents & 4 & & \\
Total & 64 & 11 & $4(5.3)$ \\
\hline
\end{tabular}

a Data are presented as No.(\%).

Table 2. Comparing Color Doppler Ultrasonography and Angiography Findings in Patients with Limb Trauma Suspicious for Arterial Injury ${ }^{\text {a }}$

\begin{tabular}{lcc}
\hline & \multicolumn{2}{c}{ Angiography Findings } \\
\hline & Normal & Abnormal \\
\hline Doppler Findings & & \\
Normal & $33(91.7)$ & $2(5.1)$ \\
Abnormal & $3(8.3)$ & $37(94.9)$ \\
\hline a Data are presented as No.(\%).
\end{tabular}


a cut-off point indicating a true and obvious arterial injury; however, this finding was reported only in 26 cases (34.7\%). Considering Doppler cutoff point, sensitivity and specificity values of Doppler USG were $66.6 \%$ and $100 \%$, respectively, and its positive and negative predictive values were $73 \%$ and $100 \%$, respectively.

\section{Discussion}

The incidence of vascular injuries has been increased worldwide during the past few decades. Motor vehicle accidents are the most common causes of injury in urban and suburban areas (8). Among different types of vascular injuries, extremity vascular injuries are one of the most critical types of limb injuries that could be life or limb threatening (6).

Arteriography as the gold standard to diagnose arterial injuries has a sensitivity of $95 \%$ to $100 \%$ and specificity of $90 \%$ to $98 \%$. Nonetheless, due to its invasive nature, it is associated with some complications in $2.6 \%$ of patients and even higher risk in patients with trauma and other coexistent injuries. Transferring a critically-ill patient to angiography ward and injecting contrast media might make the situation even worse and cause serious problems (9). Therefore, considering these special conditions, performing angiography in any multiple trauma case with findings suggestive of arterial limb injury is questionable. A recent systematic review of 51 articles showed that computed tomography angiography (CTA) is the main replacement of angiography (10). Nonetheless, color Doppler USG is nonaggressive and less expensive as a bedside method for saving patient's time and minimizing potential risks of other diagnostic interventions (7).

Bynoe et al. reported sensitivity and specificity of $95 \%$ and 99\% for Doppler USG in detection of limb vascular injuries, respectively (11). Johansen et al. and Kuzniec et al. reported similar results and according to the literature review, both sensitivity and specificity of Doppler USG to diagnose traumatic limb vascular injury are higher than 95\%, which seems acceptable for a screening and diagnostic method $(12,13)$. In our study, Doppler USG had a sensitivity of $94.8 \%$ and specificity of $91.6 \%$ to detect vascular injuries with positive and negative predictive values of 92.5\% and 94.2\%, respectively. Considering Doppler cutoff point, sensitivity and specificity of Doppler USG were $66.6 \%$ and $100 \%$, respectively, and its positive and negative predictive values were $73 \%$ and $100 \%$, respectively.

There were only three cases with abnormal findings in Doppler and normal findings in angiograms, two of which were due to an arterial spasm (one in posterior tibial artery and another in the radial artery). The third one was due to hematoma on the fractured forearm, which pushed the artery away from its normal position and therefore, the normal path of artery could not be detected well by Doppler USG, while angiography showed all the artery length and its shifting to the medial side.

There were two cases with normal findings in Doppler USG and abnormal findings in angiograms. One case had pseudo aneurysm of the distal superficial femoral artery (due to knee dislocation), which was not detected by Doppler USG because of its thick dressings. Another one was a case of cutting of proximal portion of the posterior tibial artery on angiograms, while normal wave pattern was recorded on Doppler USG because of collateral arteries. Doppler USG was not quite accurate to detect the lesion in such instances as limb splints, in situ hematoma, or patient's pain leading to lack of cooperation for optimal evaluations.

Color Doppler USG fulfilled required criteria of a screening test with a high sensitivity, acceptable specificity, availability, and being inexpensive and noninvasive; therefore, it might be considered as a suitable screening test for those with suspicious vascular injury. Nevertheless, it seems that positive results still necessitate performing an angiography. This diagnostic approach might minimize the risks of angiography in these critically ill patients.

Although Doppler USG has some diagnostic limitations in locating the exact point of artery cut-off point and the traumatized area, it would be recommended as a suitable screening test in patients with limb trauma suspicious for vascular injuries because of its high sensitivity and specificity as well as availability, inexpensiveness and noninvasiveness.

\section{Acknowledgements}

This manuscript was derived from the postgraduate thesis of Emad Ansaripour and its protocol was approved in the faculty of medicine of Mashhad University of Medical Sciences, Mashhad, Iran. We would like to thank the staff of angiography unit of Radiology Department of Imam Reza Hospital for their assistance. The authors would like to thank Dr. Mohammad Taghi Shakeri for data analysis.

\section{Authors' Contributions}

Masoud Pezeshki Rad: final approval of the article; Mahyar Mohammadifard: drafting of the manuscript; Hassan Ravari: critical revision and final approval of the manuscript for important intellectual content; Donya Farrokh: conception and design; Emad Ansaripour: provision of study materials and patients; Elena Saremi: collection and assembly of data

\section{Funding/Support}

This study was supported by the Faculty of Medicine and the Research Chancellor of Mashhad University of Medical Sciences, Mashhad, Iran.

\section{References}

1. Bravman JT, Ipaktchi K, Biffl WL, Stahel PF. Vascular injuries after minor blunt upper extremity trauma: pitfalls in the recognition and diagnosis of potential "near miss" injuries. Scand J Trauma Resusc Emerg Med. 2008;16:16.

2. Fleiter TR, Mervis S. The role of 3D-CTA in the assessment of 


\section{Pezeshki Rad M et al.}

peripheral vascular lesions in trauma patients. Eur J Radiol. 2007;64(1):92-102.

3. Lynch K, Johansen K. Can Doppler pressure measurement replace "exclusion" arteriography in the diagnosis of occult extremity arterial trauma? Ann Surg. 1991;214(6):737-41.

4. Kirkpatrick AW, Breeck K, Wong J, Hamilton DR, McBeth PB, Sawadsky B, et al. The potential of handheld trauma sonography in the air medical transport of the trauma victim. Air Med J. 2005;24(1):34-9.

5. Kohler TR, Andros G, Porter JM, Clowes A, Goldstone J, Johansen $\mathrm{K}$, et al. Can duplex scanning replace arteriography for lower extremity arterial disease? Ann Vasc Surg. 1990;4(3):280-7.

6. Reid JD, Weigelt JA, Thal ER, Francis H. Assessment of proximity of a wound to major vascular structures as an indication for arteriography. Arch Surg. 1988;123(8):942-6.

7. Meissner M, Paun M, Johansen K. Duplex scanning for arterial trauma. Am J Surg. 1991;161(5):552-5.
8. Fingerhut A, Leppaniemi AK, Androulakis GA, Archodovassilis F, Bouillon B, Cavina E, et al. The European experience with vascular injuries. Surg Clin North Am. 2002;82(1):175-88.

9. Anderson RJ, Hobson RW, 2nd, Padberg FJ, Swan KG, Lee BC, Jami $Z$, et al. Penetrating extremity trauma: identification of patients at high-risk requiring arteriography.J Vasc Surg. 1990;11(4):544-8.

10. Patterson BO, Holt PJ, Cleanthis M, Tai N, Carrell T, Loosemore TM et al. Imaging vascular trauma. BrJ Surg. 2012;99(4):494-505.

11. Bynoe RP, Miles WS, Bell RM, Greenwold DR, Sessions G, Haynes JL, et al. Noninvasive diagnosis of vascular trauma by duplex ultrasonography.J Vasc Surg. 1991;14(3):346-52.

12. Johansen K, Lynch K, Paun M, Copass M. Non-invasive vascular tests reliably exclude occult arterial trauma in injured extremities. JTrauma.1991;31(4):515-9.

13. Kuzniec S, Kauffman P, Molnár LJ, Aun R, Puech-Leao P. Diagnosis of limbs and neck arterial trauma using duplex ultrasonography. Ardiovasc Surg. 1998;6(4):358-66. 